were made by members of the conference to the Scottish Plant Breeding Station, Pentlandfield, for demonstration of genetic resistance to blight, root eelworm, seab and viruses, and the Agricultural Scientific Services Station of the Department of Agriculture and Fisheries for Scotland, for disease testing, eelworm assessment and experimental washing and disinfection. Visits were also made to the original Wedderspoon plant at Castleton, Eassie, for commercial washing and fungicidal dipping, and a store at Muthill, Crieff, where the palette system of storage is utilized, and where fungicidal dipping of cut ware tubers is carried out before planting. An informal reception by the Potato Marketing Board completed the proceedings.
Shortage of potatoes engenders public clamour and the acceptance of relatively low standards of quality: excess of supply excites less comment, but brings a fussy rejection of tubers with but slight blemish from scab or blight. Neither extreme is good, and the Potato Marketing Board has gone as far as it can by administrative means to steer between plenty and scarcity. The only valid long-term solution requires the technical discovery of an economic method of preserving surplus production for human food in times of shortage, and the Board has initiated a research programme that includes this. Such a technical solution would, moreover, contribute to the larger question of adjusting the world's food supply to its growing population.

J. Grainger

\title{
THE BIOLOGICAL ENGINEERING SOCIETY
}

$\mathrm{T}$ HE Biological Engineering Society held its second meeting of the year in the Physiological Laboratory, Cambridge, during March 23 and 24, under the chairmanship of the president, Prof. $R$. Woolmer. Sir Bryan Matthews, the guest speaker, described some engineering methods in physiology. Sir Bryan gave some instances both of the way in which apparatus has been designed at Cambridge, both past and present, to assist in teaching and research, and of the ways in which advanced engineering principles are found in biological material; the mammalian ear was invoked as an elegant example here.

P. E. K. Donaldson (Physiological Laboratory, Cambridge) described a machine for the automatic simulation of human skills. The simplest skills seem to be those of the $S \rightarrow R$ kind, in which $S$ and $R$ can each be described by a single continuous variable. Tracking tasks, and tasks involving the stabilization of unstable systems (for example, bicycle riding) require skill of this class. It has been known for some time that human operators can be represented quite closely by relationships of the form:

$$
R=e^{-p \tau}(A p+B+C / p) S
$$

Mr. Donaldson described a machine which automatically forms an image of a human operator performing a simple balancing trick, and in so doing finds values for $A, B$ and $C$ which, on the average, give the best fit. It is hoped to develop this device so as to be able to describe analytically what happens when an individual's skill improves with practice, or when it deteriorates in consequence of various kinds of distraction.

Dr. H. Barlow (Physiological Laboratory, Cambridge) discussed learning machines and generalization. Dr. Barlow felt that designers of existing learning machines, or of proposed learning machines, are trying to achieve too much too quickly. These machines can be regarded as linking units in which input data from the environment are acted on by a number of 'demons' which operate on the inputs in a variety of ways to form the outputs of the machine. Some sort of 'teacher' monitors the outputs in the light of some criteria, 'rewarding' the demons when their output is deemed valuable and 'punishing' them when it is not. Though such systems can be made to work, they are characteristically unable to do any kind of generalization, for example, to respond correctly if gaps or mistakes occur in the input data.

What is needed in order to generalize and fill gaps is knowledge of the statistics of the input. This lead, to the idea that linking units should be preceded by a 'precoder', the function of which is to form, from the raw inputs derived from the environment, new signals with known predetermined statistical properties. It is these new signals which are fed to the linking unit. Such precoding may be done by a demonflogging feedback technique, as is done in the linking unit itself, but with some important differences: the rate of feedback of information is much larger than the 1.6 bits per trial gained in the linking unit (outcome of each trial 'reward', 'punish' or 'neutral', $\log _{2} 3=1 \cdot 6$ ); the statistical requirement can be built in; and the teacher does not need to have access to the raw inputs. The output of the precoder is simpler than the raw input, since its statistics are now known, and forms a more suitable input to the linking unit.

The dynamics and operating characteristics of a common artificial kidney system were described by H. A. F. Dudley (Department of Surgery, University of Aberdeen). Dr. Dudley has been carrying out some performance measurements on an artificial kidney of the twin-coil type, the channels being $20 \mathrm{~m}$. long. The performance of such a device should be described by :

$$
D=V\left[1-\exp -\frac{P S}{V}\right]
$$

where $V$ is the rate of flow of blood, $D$ is the rate of flow of filtered blood in the output, $P$ is the permeability constant for the 'Cellophane' membrane, and $S$ the surface area of the membrane. For small values of $V$ the theoretical performance can be obtained in practice, but for higher values, which are nevertheless within the range of flow-rates possible in human subjects, the artificial kidney falls far short of what may be expected; especially if the output blood pressure is high. The discrepancy seems to be caused, at least in part, by a decline in the surface-tovolume ratio of the blood channel toward the output end. The deficiency can probably be remedied by a change in the design of these instruments; but in any event it is clear that preliminary work with models is necessary to produce an artificial kidney to meet a previously laid down specification. 
R. L. Gregory (Psychological Laboratory, Cambridge) gave a talk on "Digital Recording for the Poor Man".

It frequently happens that experimental results are recorded in a manner which retains more information than is actually required. For example, in measuring a quantity by averaging a number of determinations the mean value and the standard deviation may be important, whereas the serial order in which the readings are obtained is not. Mr. Gregory described a simple recorder he has designed in which advantage is taken of the economy possible here. A display is built up by dropping steel balls into cells in a 'Perspex' sheet or block. In one application the recorder builds up, from balls, a histogram showing the distribution of a reaction time. When the stimulus is delivered, a ball is transferred from the magazine to a moving belt, which carries it over a line of cells. When the subject responds, the ball is released and drops in the appropriate cell beneath it. The process repeats with the delivery of the next stimulus. If the reaction time is the $\operatorname{sam} \theta$, the new ball will fall into the same cell as its predecessor; in general the time will be slightly different, and the ball will fall into a neighbouring cell. Mr. Gregory pointed out how easy it should be to find the mean and standard deviation of the completed display; the former by finding where it balances on a knifeedge, and the latter by measuring its moment of inertia. So far as the display proper is concerned, there is no reason why the base of the histogram should not be some quantity other than time; the ball dropper can be positioned by an appropriate servo-mechanism. Further, the base of the histogram can be plane rather than linear, so that 'solid' models of joint frequencies may be built up.

Electrochemical recorders and memories were discussed by H. S. Wolff (Bio-engineering Laboratory, National Institute of Medical Research, Hampstead, N.W.3).

Mr. Wolff began with a disavowal; what he was going to talk about were proposals rather than achievements. He needed to produce data recorders very much smaller than those at present in general use, sufficiently small to be worn by humon subjects for long periods without causing inconvenience or discomfort. A possible solution was a small electrochemical cell possessing two anodes and one cathode.

One anode would be of simple metal, the other of a long-life radioisotope of the metal, for example, nickel and nickel-64. The quantity to be recorded would control the mark/space ratio of a multivibrator, which in turn would switch plating current between the two anodes. In this way, the quantity to be recorded would be laid down as a fluctuation in the proportion of radio nickel with the depth of the coating built up on the cathode. Such a record could be read back once, by unplating the cathode again and measuring the radioactivity of the electrolyte solution with a suitable detector.

Another problem was some way of storing a number without the continuous expenditure of energy. In contemporary analogue computing practice this is done by a servo-driven potentiometer; this technique works quite well, but is neither very small nor very cheap. An arrangement which would be both small and cheap would be to have a junction photocell with between it and a source of illumination a semi-transparent electro-plated film; the output of the photocell would depend on the thickness of the electro-deposited layer. It remained to be seen whether it would be equally satisfactory in other respects.

"Monkey Perimetry", was the subject of a paper by L. Weiskrantz (Psychological Laboratory, Cambridge). Dr. Weiskrantz and Dr. A. Cowey have been examining the effect of experimentally produced brain lesions on the field of vision of monkeys. To map the boundaries of a monkey's visual field, it is clearly not possible to use the same technique as is used with human subjects. The best that can be done is to train the monkey to respond in a recognizable way if it sees a small light flashed on; then to flash lights on, one by one, a very large number of times and from a number of different directions, recording each time where the monkey was looking. By subtracting the co-ordinates of the monkey's point of regard from the co-ordinates of the light flashed, one can-by knowing also whether the monkey saw the light or not-build up eventually a map of the monkey's perimeter of vision.

The problem was to measure where the monkey was looking. This was solved by having in front of the monkey, in addition to the field of small test lights, four bigger lights permanently switched on; one above and one below the test field in the sagittal plane, and one each side of the test field in the coronal plane. The monkey's eye is photographed during each presentation of a test light. In the photograph the four are seen reflected in the monkey's eye as four bright points arranged round the pupil. By measur. ing the distances from the bright points to the edge of the pupil, the direction of gaze can be inferred.

Unfortunately, such measurements are sensitive to translations of the monkey's eyeball as well as to rotations; but this difficulty is met by obliging the monkey to look through a peephole. The precision of each determination obtained by the technique is estimated at plus or minus two degrees of arc.

R. H.J. Brown (Zoological Laboratory, Cambridge) described the measurement of the ground reaction of the jumping locust.

Dr. Brown had carried out a rough calculation to establish the peak tension achieved in the extensor muscle of a locust's tibia when it jumps. This came out to about $1.5 \mathrm{kgm}$. and it was felt that this remarkable figure needed some experimental confirmation; the apparatus described was built in consequence.

The locust jumps off a platform stiffly mounted but capable of movement in the vertical and one horizontal direction; such movement is coupled to two capacitance transducers. In this way the transducers measure the horizontal and vertical components of the locusts's ground reaction in jumping off, effectively, a rigid platform. The damping of the mechanical system is rather below critical, and the resonance occurs at $500 \mathrm{c} . / \mathrm{s}$.; the sensitivity is $40 \mathrm{pFs}$. $/ \mathrm{kgm}$.

In the electronic gear associated with each transducer, the capacitance change alters the frequency of an inductance-capacitance oscillator and hence the voltage output of a coupled-in tuned detector circuit; this voltage is the output of the device and may conveniently be displayed on a cathode ray oscilloscope. The sensitivity is $20 \mathrm{~V}$. per $\mathrm{pF}$., giving an overall sensitivity in each of the orthogonal directions of $800 \mathrm{~V} . / \mathrm{kgm}$.

The equipment is not fully developed yet, but preliminary experiments confirm the earlier calculation; the locust can indeed produce a tension of $1.5 \mathrm{kgm}$. when it jumps.

P. E. K. Donaldson 